Original Research

\title{
Stakeholder Perceptions of Dignity Therapy for Children and Young People with Life-Limiting and Life-Threatening Conditions in the UK
}

\author{
Lucy Watts ${ }^{1}$, Joanna Smith ${ }^{2}$, Wilfred McSherry ${ }^{3,4}$, Michael Tatterton ${ }^{5}$, Alison Rodriguez ${ }^{6, *}$
}

1. Youth Ambassador Young People with Life-Limiting and Life-Threatening Conditions, School of Healthcare, University of Leeds, Leeds, UK; E-Mail: lucyalexandriawatts@hotmail.co.uk

2. Associate Professor Child Nursing, School of Healthcare, University of Leeds, Leeds, UK; E-Mail: j.e.smith1@leeds.ac.uk

3. Professor School of Nursing \& Midwifery, Staffordshire University \& University Hospitals of North Midlands NHS Trust, UK; E-Mail: w.mcsherry@staffs.ac.uk

4. Part Time Professor VID University College, Norway

5. Nurse Consultant Martin House Hospice, Wetherby, UK; E-Mail: mtatterton@martinhouse.org.uk

6. Lecturer Child and Family Health, School of Healthcare, University of Leeds, Leeds, UK; E-Mail: a.m.rodriguez@leeds.ac.uk

* Correspondence: Alison Rodriguez; E-Mail: a.m.rodriguez@leeds.ac.uk

Academic Editor: Leila Kozak

Special Issue: Integrative Therapies in Palliative Care

$O B M$ Integrative and Complementary Medicine 2020 , volume 5 , issue 1

doi:10.21926/obm.icm.2001012
Received: November 04, 2019

Accepted: February 25, 2020

Published: March 03, 2020

\begin{abstract}
In palliative and end of life care settings supporting people to make sense of their lives is as important as managing disease symptoms. Dignity Therapy is a validated psychotherapeutic intervention designed to bring about a sense of meaning and purpose for individuals at the end of life. Dignity Therapy has primarily been implemented and evaluated in adult palliative care settings. Prior to designing studies that evaluate Dignity Therapy for children and young people with life-limiting and life-threatening conditions, it is essential to establish stakeholders' perceptions of Dignity Therapy, and whether adaptations would be required
\end{abstract}

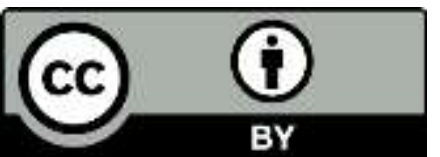

(C) 2020 by the author. This is an open access article distributed under the conditions of the Creative Commons by Attribution License, which permits unrestricted use, distribution, and reproduction in any medium or format, provided the original work is correctly cited. 
for this client group. We aimed to explore the acceptability of Dignity Therapy for children and young people with life-limiting and life-threatening conditions and health professionals, and report findings from our stakeholder activities. Stakeholder consultation activities involved one Death Café and two workshops, with over 80 health professionals, hosted at two hospice settings, and a range of social media activities including feedback from five young people with life-limiting and life-threatening conditions via a closed Facebook group and a twitter chat with 22 contributors. Data included field notes, workshop outputs ('post-it ideas storm' and 'diamond ranking') and social media postings that were analysed using thematic analysis. Across stakeholders, there was overwhelming support for explicit interventions that support the psychosocial and spiritual needs of children and young people with life-limiting conditions. Dignity Therapy could be a supportive intervention to promote open conversations about life, meaning and purpose and dying, between the child or young person, family members and health professionals, but needs adapting to meet their needs. A tool kit of interventions, appropriate to a range of ages and cognitive abilities was suggested, for example a storybook for young children and a digitalised version of dignity therapy for older children/young people. Collaboration with stakeholders is the first stage in developing, or adapting an existing intervention in a different context, a complex intervention. Our stakeholder consultation highlighted that Dignity Therapy has potential to improve the psychosocial and spiritual wellbeing of children and young people with life-limiting and lifethreatening conditions, through recalling memories and thinking about the things that are important to them and what they want to be remember for. Research is now needed to develop and test a Dignity Therapy intervention for children and young people with lifeliming and life-threatening conditions in the UK.

\section{Keywords}

Dignity therapy; stakeholder involvement; life-limiting conditions

\section{Background}

Dignity Therapy (DT) is an evidence-based psycho-therapeutic intervention that has been found to improve the psycho-social and spiritual wellbeing of adults with life-limiting and life-threatening conditions (LLTC), through a process of life-review and meaning-making $[1,2]$. DT involves supporting patients to discuss their valued memories, accomplishments, life lessons and hopes for significant others. The intervention aims to bring about a sense of meaning and purpose for patients at the end of life, and their families [3].

DT emerged from the Dignity Conserving Model of Care (DCMC); this model was derived from qualitative studies undertaken with adult cancer patients receiving palliative care [4]. During DT, trained professionals undertake one-to-one semi structured interviews with individual patients, usually lasting for about an hour. The interview is structured by drawing on nine questions that relate to facets of the DCMC. This set of questions is referred to as the Dignity Therapy Questionnaire Protocol (DTQP) [1, 4]. 
The DT interview is digitally recorded, transcribed, edited and returned to the patient, who can then make any changes before the document, called a generativity document or written legacy, is finalised. Many patients share these documents with significant others prior to their death, providing opportunity for meaningful conversations that can assist bereavement processes [5]. This person-centred therapeutic approach can also help health professionals understand individuals in the wider context of their lives and tailor support to their needs [6].

The growing evidence-base of DT in adult palliative care settings suggests that DT is more likely to improve spiritual wellbeing, overall quality of life, hope and sadness/depression scores that standard care alone [7-11], and has also been positively received by family members [12]. Health professionals report DT is a brief and flexible intervention that can be integrated into established supportive palliative care practices [12].

\section{Introduction}

There are over 50,000 children and young people (CYP) living with a life-limiting condition or life-threatening (LLTC) in the UK, which is predicted to increase with technological and medical advancements [13]. While these advancements have resulted in better management of these conditions, there remains uncertainty around prognosis, with many CYP living shortened lives [13]. The impact of LLTC on CYP, parents, siblings and the wider family is significant in terms of their emotional wellbeing [14, 15]. Improving wellbeing throughout their shortened life journey, especially towards the end of life, requires care that addresses their psycho-social and spiritual needs, but this can be challenging for health professionals [16].

Palliative care for CYP supports the social, psychological and spiritual needs of CYP with expected shortened lives and their families $[8,15]$. However, there remains a significant unmet need in relation to meeting the psycho-social and spiritual wellbeing of CYP with LLTC, acknowledging their meaning-making and generativity needs and heightened risk of mental health problems $[17,18]$. Supporting CYP with LLTC to cope with their condition and integrating tailored psycho-social and spiritual support into care pathways could improve mental health and wellbeing outcomes $[13,17]$.

Our evidence synthesis of published research focusing on CYP (aged up to 24 years) with LLTC found only four published studies about DT and related meaning making interventions for this age group [17]; one focused on young people (7-17 years) [19], the other three included young people but mean ages were 50-70 years $[2,3,20]$. In summary, and while recognising the limited evidence-base, we identified:

1. DT has potential to improve psycho-social wellbeing, emotional functioning, dignity and hope for CYP with LLTC;

2. DT is unique, welcomed and perceived as helpful for CYP and the family;

3. DT has not been widely used or evaluated specifically for CYP with LLTC. The studies that have included CYP have largely focused on older children / young people with cancer;

4. No UK or European studies were identified that had evaluated the acceptability, feasibility and effectiveness of DT or related meaning making interventions for CYP with LLTC.

Our review also highlighted missed opportunities to support CYP with LLTC and their families to engage in meaningful discussion about dignity, meaning making and end of life care in a structured way. 
Since the publication of our review, Juliao et al [21] have published their work on adapting the DTQP for adolescent's aged 10 to 18 years in Portugal. They have developed an amended DT set of questions which could be used in practice for sick Portuguese adolescents. Although a five-stage process of validation is detailed, the amended DTQP is not provided and so it is unclear as to whether the new question format is aimed at those with typical/atypical cognitive development and for what conditions. At this stage we therefore cannot be sure if the proposed DT is appropriate for CYP in the UK with LLTC.

We have brought together expertise in advocacy for CYP with LLTC, palliative care, psychotherapy, children's nursing and spiritual care, to consider how to adapt and develop DT to produce an intervention/s that would be appropriate for CYP with LLTC in the UK.

We have also determined that stake holder consultation needs to be central to the proposal of amending/developing DT for CYP with LLTC for a number of reasons; 1 ) It is a moral obligation that healthcare delivery and research is responsive and addresses patient or in this case CYP / family priorities; 2) The sensitivity required when delivering DT type interventions must consider the views of CYP, their families and health professionals delivering care; 3 ) Involvement must not be tokenistic to meet funding body requirement; 4) The design and methods of undertaking DT research are appropriate for CYP with LLTC; 5) Stakeholder involvement is vital to shape and ensure the patient voice remains the focus of the research, even in the early stages of developing a programme of research.

The lead author (LW) is a core member of the MAGICYL team, and a young adult with a lifelimiting condition who has been involved in this study from its inception, co-designing our activities including preparing participant information and collecting information from young people.

The broad aim of our stakeholder activities was to explore whether DT would be acceptable for CYP with LLTC and health professionals.

The specific objectives were to:

1. Explore CYP with LLTC views of DT;

2. Explore health professionals, who support CYP with LLTC, perceptions of DT for this patient group;

3. Identify what, if any, meaning making activities are used in clinical practice for CYP with LLTC;

4. Review the DTQP and consider its application for CYP with LLTC.

\section{Materials and Methods}

We followed the National Research Ethics Service (NRES) and INVOLVE guidance [22], which states that ethical approval is not needed where people are acting as specialist advisers, providing valuable knowledge and expertise based on their experiences. However, in keeping with good practice, we obtained written consent from participants of the death café and closed Facebook group and verbal consent from workshop participants and have only included data if permission was given. For the twitter chat, participation was assumed if people contributed. All participants were above 16 years of age. 
AR, PI of the research team has completed the Dignity Therapy training course, undertaken in Canada. In addition, permissions were sought and received from the founder of DT, Prof Harvey Max Chochinov to explore amending and developing DT for CYP with LLTC in the UK.

Our health professional consultation experts were drawn from two children's hospices in the North of England, that provide family led care for CYP with LLTC. Our social media activities were not geographically restricted and included anyone with an interest in improving the psycho-social and spiritual wellbeing for people with LLTC and included young people. We undertook a range of consultation activities, in summary:

1. A Death Café was hosted by one of the hospices, facilitated by AR and JS, with a total 9 participants; 1 art therapist, 1 psychologist, 5 children's nurses, 1 psychologist, 1 adult nurse.

A Death Café is a directed discussion about death rather than a support group, and while public facing cafés have no agenda or objectives, Death Café type groups are increasingly being offered as a support mechanism for health professionals (Nelson, 2018). The first hour of the Death Café was unstructured giving participants opportunity to talk if they wished, or just listen to the conversations about death and dying. In the second hour DT was introduced, and participants were guided to discuss their views of the concept of DT and its possible application to CYP with LLTC.

2. Our social media activities involved:

1) Asking young people via a closed Facebook account, that LW established, whether DT could be relevant to them, they shared their views in a short-written narrative; 5 individuals with LLTC participated, aged $16-24$ years.

2) We hosted a Twitter chat, for one hour, that involved tweeting a series of questions and responding to tweets to generate an online debate and capture broader views about DT and its relevance to CYP with LLTC. We summarised the chat in an online story that enabled us to include postings in subsequent data analysis [23]. Twenty-two individuals who engaged in the chat identified themselves; 8 children's nurses, 2 adult nurses, 6 Psychologists, 3 young people with LLTC and 2 parents of CYP with LLTC.

3. Two workshops were hosted at the second hospice with a total of 45/46 participants in each workshop; 38 children's nurses, 9 learning disability nurses, 5 adult nurses, 5 physiotherapists, 3 music/art therapists, 2 social workers, 1 occupational therapist, 1 psychologist, 30 support workers.

Two tasks were undertaken:

1) The first included 'post-it ideas storm', 'dot voting' and 'diamond ranking' from 'Participation - spice it up: a practical tool' [24] to identify what activities, whether labelled as DT or not, health professionals who support CYP with LLTC undertake that focus on end of life issues, and to maximise everyone's contributions. The process involved everyone writing down the activities they used, or thought would be useful on a separate post-it and placed them on a wall ('post-it ideas storm'). 'Dot voting' was used to gain a consensus about which activities would be most useful or could be delivered in practice; participants were given 3 sticker dots each and asked to place their dots next to those activities they considered most important or relevant to DT. 'Diamond Ranking' involved placing the post-its on a large diamond, grouping similar activities topics together and placing those identified as most relevant (through dot voting) at the top of the diamond and those least relevant at the bottom. Through discussion and negotiation, a consensus of possible activities that might support DT type interventions were identified. We photographed 
the 'diamond ranking' collage from both groups so that the data could be incorporated into our analysis.

2) Each participant was provided with the DTQP and either individually or if they preferred working in pairs were asked to consider each question and document their thoughts in relation to whether the question was suitable for CYP with LLTC, and if not how could be adapted.

The principles of thematic analysis were used to bring the data sets together, describe and summarise our activities [25]. First, AR typed the field notes and activities into a usable format; a word document that include both narratives and tables. AR and JS read the data sets and began the process of grouping data together, initially using the consultation objectives as a guide. Gradually as the different data were analysed categories emerged. Categories were modified as new insights became apparent until a coherent account developed that represented all data sets. Categories were grouped into two overarching themes. Bias was reduced by ongoing refinement of categories and discussions between all authors at three team meetings.

Participants in the Death Café and closed Facebook group were given a shopping voucher as a thank you for their time, with participants at the workshop given a thank you gift. This did not influence participants' decisions to contribute as they were unaware of the gift in advance, but we wanted to recognise their willingness to share their insights, experiences, and being involved in open discussions about a sensitive topic and area of care.

\section{Results}

One hundred and twenty-one people participated in our consultation events. Analysis of the data highlighted that both CYP and health professionals' perceived DT was highly relevant for CYP with LLTC but that discussing death and dying often lacked focus and could be particularly challenging when working with young children.

Our stakeholder participants overwhelmingly supported the relevance of a DT type intervention/s for CYP with LLTC:

'it is asking the questions that need to be asked' (Workshop participant).

'l love the idea that it's something to remember me by and something that would

hold everything I think that's happened to make my life amazing' (Facebook participant).

However, a DT for CYP with LLTC needs to flexible to meet a range of ages, cognitive and physical disabilities to be embedded into practice. The two themes and associated categories that emerged from the analysis are presented in Table 1.

Table 1 DT for CYP with LLCs: themes and categories.

\begin{tabular}{|c|c|}
\hline Themes & Categories \\
\hline $\begin{array}{l}\text { Meeting the psycho-social } \\
\text { needs of CYP with LLTC }\end{array}$ & $\begin{array}{l}\text { - Making DT meaningful for CYP with LLTC } \\
\text { - Empowering young people }\end{array}$ \\
\hline Embedding DT into practice & $\begin{array}{l}\text { - Building DT into current practice } \\
\text { - Support for health professional }\end{array}$ \\
\hline
\end{tabular}




\subsection{Meeting the Psycho-Social Needs of CYP with LLTC}

Across stakeholder participant groups, there was overwhelming support for explicit interventions that support the psycho-social needs of CYP with LLTC. While 'legacy leaving' type activities are already undertaken, for example through developing memory boxes, the purpose of the activity is implicit; greater transparency may facilitate conversations about death and dying developing.

Participants perceived that there remains an unwillingness to talk about death and dying, particularly with CYP, as the follow account highlights:

'There is a fear of death in general and to a degree, those who work within palliative care and especially with children. Although dying is an end point for us all, palliative care is about quality of life for those with shortened lives', (Death café participant).

'The hospice supports children who are not expected to live beyond 19 years. For those whose prognosis may be more favourable or unknown, very little can be offered. Being able to offer Dignity Therapy could offer some level of psychosocial support to this group', (Death café participant).

'It is very hard to live with a life-limiting illness and Dignity Therapy seems to focus on giving validity to the life of someone with these types of illness. Looking back to see what made them happy and what made their life fulfilled. Not just focusing on hospital and medical stuff which can take over the lives of people with these illnesses', (Facebook participant).

Our stakeholder participants perceived DT could promote open conversations about dying and had the potential to capture memories for CYP with LLTC and could therefore improve their overall wellbeing. However, DT interventions and output for example the generativity document, need to be meaningful for CYP with LLTC and using their words.

\subsubsection{Making DT Meaningful for CYP with LLC}

Our PPI participants highlighted that DT for CYP with LLTC needs to meet a diverse range of ages and stages of cognitive development, skills and abilities, and especially for CYP with difficulties communicating. For example:

'Children with neurological deterioration can have significant changes in personality and holistic functioning. We are often reliant on the narratives of families to know what the child is like to build their stories through rehabilitation or palliative care', (Death café participant).

'We need to think about those who cannot communicate or only have a few words', (Workshop participant).

For DT to become established as part of care for all CYP with LLTC a range of activities would need to be developed that are user friendly (for both CYP and those delivering the activity), and be relevant to the CYP, such as using digital mediums for young people and creative arts for younger children. There was overwhelming support for developing 'a tool-box' of activities:

'Dignity Therapy could form the basis of a toolbox of activities for this group', (Death café participant). 
I think depending on the age/experience/illness of the child, would depend on what approach you would use. I think anyone who is facing death, they would want to know that someone is going to remember them, and something made their life worthwhile. If someone has been diagnosed with a terminal illness that is going to take them quickly, they will need an approach that fits with their time scale'. (Facebook participant).

Workshop participants, through the 'Diamond Ranking' task, identified a range of activities that could have a DT component, many using digital mediums (Figure 1).

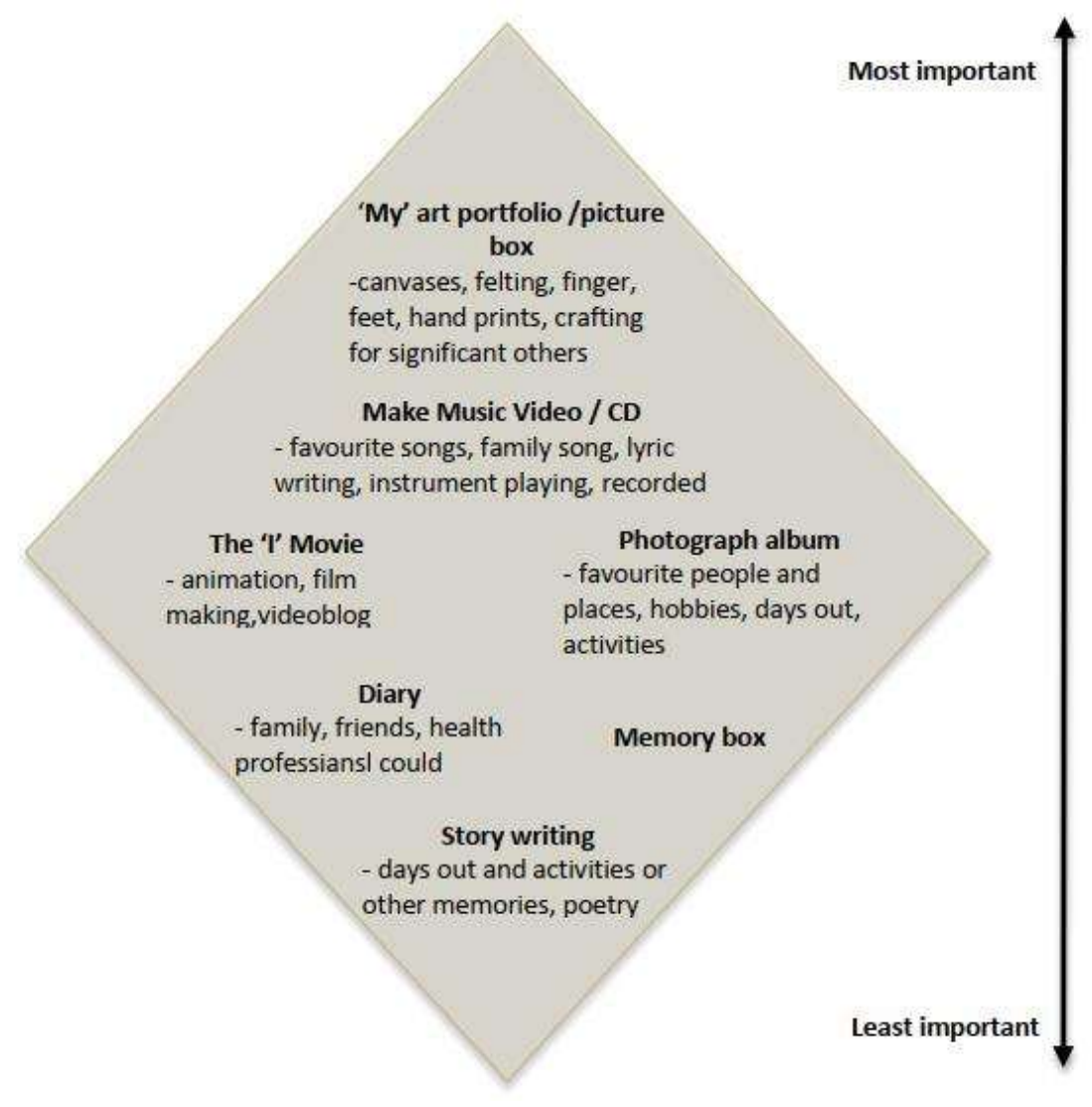

Figure 1 Diamond rank of meaning making activities.

Stakeholder participants perceived that for some CYP with LLTC there may have been limited opportunities to fulfil their wishes and hopes and suggested that through DT a range of memory making activities could be identified, planned and captured, for example days out / experiences. Participant across data sets highlighted that the illness trajectory for CYP with LLTC is more uncertain than in adults populations, and therefore DT type interventions must be adaptable across the LLTC trajectories of individuals, be flexible and revisited at different transition stages, rather than an intervention that is delivered as a time bound task. Examples of participants accounts of DT for CYP with LLTC included shared sentiments:

'Lots of our young people have not had opportunities to explore 'roles', some have very limited life experiences, most but not all', (Workshop participant).

'Dignity Therapy could be adapted to be delivered at different age stages/transitions of care/stages of disease trajectory', (Death café participant). 
'Dignity Therapy could be the basis for a supportive intervention for CYP not just at the end of life in palliative care but leading up to (if diagnosis is difficult) or shortly following a life threatening/limiting diagnosis and thereafter', (Death café participant).

Sixty-five workshop participants (noting some participants worked in pairs) commented on the DTQP, only one person reported that the DTQP was unsuitable for CYP and made no suggestions in relation to adapting the questions. However, overall, there was support for the general intent of the questions:

'With rewording a little this is a good supportive intervention, it would need sensitivity and courage to do', (Workshop participant).

Workshop stakeholder participants highlighted that DTQP could be more meaningful for CYP, and there needed to be greater flexibility for health professionals when working with the younger age group and for CYP with communication difficulties. A shared concern was the use of the 'past tense' in the original DTQP which was perceived as not useful / lacked sensitivity (Table 2), when considering the uncertain illness trajectory for many CYP with LLTC. A range of alternative phrasing was offered, with some terms such as 'role' argued to be too adult focused:

'Role could be taken as demoralising if they feel they have not achieved much', (Workshop participant).

'I think it would be good to adapt it slightly for young people to make it more suitable to the current day, for example incorporating modern technology. I think using a "vlogging" type system may be good to record special memories or thoughts which could then make a larger video. Another way would be to use social media more', (Facebook participant).

... for it (DT) to include different things at different points in time, (Facebook participant).

Table 2 summarises the second task undertaken by workshop participants which was to consider each question of DT. There was a consensus that some of the questions could be difficult to understand for younger children and for young people with communication difficulties and complex needs. For example, CYP are likely to struggle to appreciate the difference between questions three and four. However, questions five to nine were highlighted as highly relevant and could be discussed through creative methods/activities. There was also further consideration that the intervention could be digitised for CYP:

'Questions could be centred on creative activities especially for those none verbal or with complex needs', (Workshop participant).

'Is this something that could be supported by technology?', (Workshop participant).

'What I feel would work really well is if there was a D T legacy website. Many forms of social media have become an online outlet for people of all ages and walks of life... The individual then has peace of mind that their legacy is not able to be lost or damaged, it is digitally available to be shared with all those you wish to see it, living on through a legacy that can remain for many years to come and can be downloaded by the family to keep offline if they would like to do so'. (Facebook participant). 
Table 2 DCMC Therapy Questionnaire Protocol (DTQP) and suggested adaptations.

\begin{tabular}{|c|c|c|}
\hline DCMC Therapy Questions & Comments/suggested changes & Adapted questions \\
\hline $\begin{array}{l}\text { 1. Tell me a little about your life } \\
\text { history, particularly the parts } \\
\text { that you either remember } \\
\text { most, or think are the most } \\
\text { important. } \\
\text { When did you feel most alive? }\end{array}$ & $\begin{array}{l}\text { Participants did not perceive 'when did you feel most alive' as appropriate for } \\
\text { CYP, some felt it was too adult or too formal, some thought the question was } \\
\text { inappropriate and that it should be removed. } \\
\text { Others considered phrasing the question in the present would be better - the } \\
\text { past is negative. Using the term 'story' would also be more appropriate than life } \\
\text { history } \\
\text { Suggestions included: } \\
\text { Tell me a little about your story/ tell me about your life } \\
\text { What inspires you? When do you feel most happy? } \\
\text { What are your highlights so far? } \\
\text { What are your } 3 \text { most memorable moments? } \\
\text { What makes you most happy/vibrant/exhilarated? }\end{array}$ & $\begin{array}{l}\text { 1. Tell me a little about you / your story, } \\
\quad \text { when do you feel most happy? } \\
\text { Prompts: } \\
\text { What are your } 3 \text { most memorable } \\
\text { moments? } \\
\text { When did you feel at your best? } \\
\text { When were you the happiest? } \\
\text { What inspires you? }\end{array}$ \\
\hline $\begin{array}{l}\text { 2. Are there specific things that } \\
\text { you would want your family to } \\
\text { know about you, and are there } \\
\text { particular things you would } \\
\text { want them to remember? }\end{array}$ & $\begin{array}{l}\text { Participants perceived this question with slight adaptation to be highly relevant } \\
\text { but that for some CYP, thinking about 'after' is difficult. } \\
\text { Suggestions included: } \\
\text { How would you like to be remembered? } \\
\text { How do you think your family/friends will remember you? } \\
\text { What do you think your parents/friends will remember about you? }\end{array}$ & $\begin{array}{l}\text { 2. What do you think your parents/friends } \\
\text { will remember about you? } \\
\text { Prompts: } \\
\text { How would you like to be remembered? } \\
\text { Are there things you would not want your } \\
\text { family to know? }\end{array}$ \\
\hline
\end{tabular}


3. What are the most important roles you have played in life (family roles, vocational roles, community service roles, etc.)? Why were they so important to you, and what do you think you accomplished in those roles?
Again, participants highlighted that questions needed to be in the present for example 'play' rather than 'played' as life is not over yet. Some CYP are not selfaware or confident, some might say nothing and may have had very limited life experiences /opportunities to explore 'roles.' 'Role' could also be taken as demoralizing if they feel they have not achieved much.

This question was also perceived as 'a burden to parents and siblings'. In addition, some CYP have their conditions all their lives, which can make this question difficult and is probably not needed or needs simplifying.

\section{Suggestions included:}

What have been your proudest moments?

What are your accomplishments? This could be integrated with question 4.

What things are you most proud of and why? Although some CYP may struggle with articulating this and may feel they have none.

What are the best things that you have taken part in or have done in your life? Instead of roles, say issues or things such as friendships, having a dog.

4. What are your most important accomplishments, and what do you feel most proud of?
The majority of participates perceived CYP, particularly younger children, would struggle answering this question and it could cause distress if they feel they have no accomplishments.

One participant suggested that this question is a 'little scary' when thinking about how it would be asked.

Thinking about working with CYP, questions 3 and 4 could probably be adapted by health professional and could be combined.

\section{Suggestions included:}

Simply ask, of all the things you have done, what do you feel most proud of?

What has made you happy? What are your happiest memories?

Is there anything you want to share with your family?

\section{What are the best things that you have} taken part in or have done in your life?

This question combines question 3 and 4 of the original DTQP

Prompts:

What do you feel most proud of? What has made you happy?

Are there things you would want people to know about you? Or tell people close to you?

Are there things you want to do?

Do you need any help to say/write these? 
5. Are there particular things that you feel still need to be said to your loved ones, or things that you would want to take the time to say once again?

6. What are your hopes and dreams for your loved ones?

7. What have you learned about life that you would want to pass along to others? What advice or words of guidance would you wish to pass along to your (son, daughter, husband, wife, parents, others)?
Participants perceived this question, with adaptations, to be highly relevant because 'we shy away from this question and in doing so we are being overprotective'.

However, consensus surrounded how the wording perhaps needs adapting, for example to not use the term 'loved ones', and instead say 'friends and family'.

Suggestions included:

Is there anything you want to say to the people you care about?

Are there things you would want people to know about you? /Do you need any help to say/write these?

Are there things you want to do?

Although there was much support for this question, participants thought it may be difficulty for CYP to articulate when answering.

Suggestions included:

Do not use the term 'loved ones', replace with 'friends and family'.

Do you have any hopes for the people close to you?

Language is very adult orientated, but it was agreed that this is an important question.

Change items in brackets to just friends and family, and the question could be clearer.

Question 7 and 8 could be combined, as they seem to be asked similar things. Suggestions included:

Is there anything you would like to pass on to your loved ones before you die? (Be honest, you can be direct without being insensitive).

What advice or words of guidance would you wish to pass along to your friends and family?
4. Is there anything you want to say or repeat to the people you care about? Prompts:

Your family?

Your friends?

Do you need any help to say/write these?

Others?

5. Do you have any hopes for the people close to you?

\section{What advice or words of guidance} would you wish to pass to your friends and family?

Prompts:

Is there anything you would like to pass on to family and / or friends before you die? 
8. Are there words or perhaps even instructions you would like to offer your family to help prepare them for the future?

9. In creating this permanent record, are there other things that you would like included?
There was great support for this question, but it was perceived that it could be a burden for some CYP.

More suitable for older children.

Question 7 and 8 could be combined, seem to be asked similar things, make clear purpose of question

\section{Suggestions included:}

Are there any words or perhaps instructions you would like to offer your family to help prepare them for life without you?

Participants perceived this question, with adaptations, was highly relevant

Remove 'permanent' - it is highly likely that CYP would want to change and edit their document over time.

Art ideas and memory boxes could fulfill this question.

A good opportunity to make a digital record, which could be multimodal using arts and media such as music!

\section{Suggestions included:}

Is there anything that needs to be included that we have not talked about?

Can we create a special time for you? A special day with your family?

There could be scope with CYP with LLTC to build on responses to question 9 and engage them in focused DT influenced creative arts, activities, related discussions, to contribute to what could be a DT project for the individual.
7. Are there any words or perhaps instructions you would like to offer your family to help prepare them for life without you?

\section{Is there anything that needs to be} included that we have not talked about?

\section{Can we create a special time/activity for} you?

This question is an addition to the original DT protocol.

For example, and within local limits -

A special day with your family.

Creating music work, with you and your family.

Creating artwork, with you and your family. Followed by memories captured of the day 


\subsubsection{Empowering Young People}

Our stakeholder participants perceived DT type interventions could promote open conversations about dying and had the potential to capture memories for CYP with LLTC, and empower them to share their hopes, fears and concerns.

'This could be a very beneficial tool and would empower CYP', (Workshop participant).

'Giving people permission and broaching sensitive subjects are powerful things.

People can be so relieved to just be asked the question \#ebnjc,' (Twitter chat participant).

I think I would want to do it alone so that I can express my true feelings without being influenced by the thoughts or emotions of my family. Also then it would be a surprise to them when completed, (Facebook participant).

'One thing that people don't talk about on social media quite so openly is more challenging subjects such as death, loss, illness and all that comes with it. That's why I feel that having a website tailored to D T legacies it would remove that dark cloud that hangs over these topics' (Facebook participant).

DT is viewed by our stakeholder participants to be an activity that could support CYP with LLTC to broach challenging conversations and to allow them to have some level of control over what they want to share, at what time and with whom. DT is a supported intervention whereby professionals could be led in their supportive practices by the CYP, acknowledging what the CYP has experienced as meaningful in their lives lived so far and in considering what their hopes or concerns may be for the future.

\subsection{Embedding DT into Practice}

Some of the DT type interventions already described are delivered in practice but are not aligned to a DT based or meaning making model/intervention. For example, there may be reflection or memory making activities undertaken in hospice settings, but these are usually undertaken just as creative and enjoyable events. Often activities are undertaken without consideration of broader meaning making issues or discussion over how the activity may contribute to legacy, directly with the CYP. In consideration of how DT could become a tool for CYP palliative and psychotherapeutic care, our stakeholder participants offered comments that can allow us to consider how we could integrate DT into current practice and what support health professionals would need themselves to engage with DT.

\subsubsection{Building DT into Current Practice}

DT in its established face to face, interview format was viewed as daunting and inappropriate for all CYP with LLTC, from the perspective of health professionals.

I am hesitant about any face-to-face questioning and formal presentation. All the most meaningful, inner most thought discussions I have had with young people have never come out in questionnaire type conversation, but rather when 
doing daily activities such as in a car, bathing, putting to bed, art activities etc., so use as a distraction from full on pressured responses', (Workshop participant).

DT, however, still allows the CYP to lead conversations and to have ownership over what they want to reflect upon and share/discuss.

'This is fascinating and I can see how DT could enhance CYP limited lives, value who they are and maybe strengthen relationships \#ebnjc', (Twitter chat participant).

As aforementioned, it was felt that the re-framing of some questions would benefit CYP with LLTC, given their lives lived may be very different to older adults with advanced conditions. With this possibility and a clearer understanding of how different and existent activities could fit into the framework of DT, professionals were enthusiastic of the possibilities and potentials of DT being integrated into practice.

\subsubsection{Support for Health Professional}

CYP with LLTC can have a range of conditions and needs, with varied communication/cognitive abilities and very different disease trajectories. This necessitates the development of DT to be flexible to individual CYP needs. A toolbox of DT activities that includes digital applications, creative activities and supportive discussion protocols may be most appropriate for health care professionals and CYP with varied needs to access.

I imagine that's a big problem with getting it into practice - a lot of groundwork needs to be done to ensure CYP can access DT \#ebnjc', (Twitter chat participant). 'Unlike adults, young people's minds are changing dramatically in relatively short amounts of time and for that reason their understanding of life, themselves and their surroundings will constantly evolve. Their opinions thought patterns and core beliefs may also change dramatically as they gain more understanding of society with age and their relationships with loved ones and their desires for their own lives will also be in constant development. For this reason, I feel it is important that a young person would have time to build on their $D T$ legacy so that as they change as a person it can be reflected through their legacy' (Facebook participant).

Once DT is amended and developed for CYP it would be appropriate for trained DT therapists and the researchers developing DT for CYP with LLTC to then consider a programme of training to support health care professionals to deliver and monitor DT and its effects.

'Practitioners would need to understand the principles of DT and be trained in the application of DT the intervention', (Workshop participant).

'Practitioners would also need where appropriate, to be able to assist CYP to be involved in creative arts, (Workshop participant).

DT is an evidenced based psychotherapeutic intervention that requires training to appreciate its underpinnings and to consider ways to best support individuals in life review, meaning making and legacy leaving. The generativity documents/legacies created need to support the needs of the CYP with LLTC but also be supportive to recipients. 


\section{Discussion}

Our findings suggest DT has the potential, with adaptations, to support CYP with LLTC and improve their overall wellbeing and quality of life. DT type interventions need to be developed and evaluated specifically for CYP with LLTC in the UK. The DTQP and related activities need to be responsive to the uncertainty of conditions and be appropriate to a range of ages and cognitive abilities.

Meaningful discussion about dignity, legacy and end of life care are currently being missed in CYP palliative care because activities are not identified as interventions to support the psychosocial and spiritual needs of CYP with LLTC facing shortened lives [26]. The DCMC hypothesises that there is a reciprocal interaction between aspects of human experience in DT which alleviates psycho-social and spiritual burdens $[4,6]$ which is highly appropriate for CYP with LLTC. Meeting the spiritual and existential needs of CYP with LLTC is likely to help them cope with their condition and develop resilience to meet life challenges [27, 28].

Our stakeholder consultation work suggests that there is scope to create a toolbox of DT activities for CYP with LLTC. DT generativity documents are being discussed in the literature and are evolving; for example, DT with older adults has resulted in producing picture books, including cherished photographs alongside key commentaries [29] and digital storytelling has shown to be beneficial with CYP with cancer conditions [19]. The stakeholder consultation findings highlight that for many CYP with LLTC that the generativity output would need to be updated: a digitalized format would provide CYP with LLTC the scope to edit and change and to save content and rethink and add at different junctures in their illness trajectories.

Many CYP with LLTC reach a crisis in terms of physical deterioration in adolescence or early adulthood, yet their psycho-social and spiritual needs are not currently addressed adequately [13]. CYP with LLTC facing impending death also need opportunities to explore their feelings without fear of upsetting family members [15, 30, 31].

We recognise that CYP with LLTC have unique physical and psycho-social needs, many rely on parents as their main source of emotional support and to undertake tasks of daily living [13]. DT would support CYP with LLTC if they wished, to share experiences and wishes without reliance on their parents or significant others. DT would allow CYP with LLTC to bequeath material they feel is important to share but may be too emotionally difficult to discuss with loved ones directly.

\section{Conclusions}

We have explored the views of young people with LLTC, parents and health professionals about DT and its relevance as a supported intervention for CYP with LLTC. Our stakeholder participants support the amendment and development of DT for CYP with LLTC. The key conclusions achieved following these 18 months of preparatory work include:

1) DT could be a supportive and supported intervention for CYP with LLTC;

2) There is potential psycho-social and spiritual benefit to CYP with LLTC recalling memories, imparting advice and considering meaningfulness through DT;

3) DT could promote open conversations about dying between the CYP with LLTC, family members and HCP; 
4) For CYP with LLTC whose prognosis may be more favourable or unknown, little can be offered by hospice care. Being able to offer DT could therefore be invaluable for this group;

5) CYP with LLTC have lived most, if not all their lives in the technological age, therefore they may be more willing or motivated to engage in a digitalised DT;

6) There is consensus that DCMC is suitable for CYP with LLTC, but the phrasing of some questions of the DTQP could be more appropriate for CYP with LLTC;

7) An amended DTQP for CYP with LLTC is suggested; the DTQP must be rigorously refined so that it retains the original purpose and is meaningful and relevant to CYP with LLTC.

8) A toolbox of DT based activities would be supportive for both HCP and CYP with LLTC in practice.

Our next step is to take forward the findings of these stakeholder consultation activities and begin to plan studies that will focus on the amendment and development of DT for CYP with LLTC and to test their feasibility.

\section{Acknowledgments}

We thank Prof Max Harvey Chochinov for supporting our venture to explore Dignity Therapy for CYP with LLC in the UK.

\section{Author Contributions}

All authors contributed to the content of consultation activities. Consultation activities were undertaken by AR, LW, MT and JS. AR and JS completed the analysis and all authors then contributed to the development of this paper.

\section{Competing Interests}

The authors have declared that no competing interests exist.

\section{References}

1. Chochinov HM, Hassard T, McClement S, Hack T, Kristjanson $L$, Harlos $M$, et al. The patient dignity inventory: A novel way of measuring dignity-related distress in palliative care. J Pain Symptom Manage. 2008; 36: 559-571.

2. Chochinov HM, Kristjanson LJ, Breitbart W, McClement S, Hack TF, Hassard T, et al. Effect of dignity therapy on distress and end-of-life experience in terminally ill patients: A randomised controlled trial. Lancet Oncol. 2011; 12: 753-762.

3. Chochinov HM, Hack T, Hassard T, Kristjanson LJ, McClement S, Harlos M. Dignity therapy: A novel psychotherapeutic intervention for patients near the end of life. J Clin Oncol. 2005; 23: 5520-5525.

4. Chochinov HM. Dignity-conserving care-a new model for palliative care: Helping the patient feel valued. JAMA. 2002; 287: 2253-2260.

5. McClement S, Chochinov HM, Hack T, Hassard T, Kristjanson LJ, Harlos M. Dignity therapy: Family member perspectives. J Palliat Med. 2007; 10: 1076-1082.

6. Chochinov HM. Dignity therapy: Final words for final days. Oxford: Oxford University; 2012. 
7. Dose AM, Rhudy LM. Perspectives of newly diagnosed advanced cancer patients receiving dignity therapy during cancer treatment. Support Care Cancer. 2018; 26: 187-195.

8. Hall S, Edmonds P, Harding R, Chochinov H, Higginson IJ. Assessing the feasibility, acceptability and potential effectiveness of Dignity Therapy for people with advanced cancer referred to a hospital-based palliative care team: Study protocol. BMC Palliat Care. 2009; 8: 5.

9. Juliao M, Oliveira F, Nunes B, Vaz Carneiro A, Barbosa A. Efficacy of dignity therapy on depression and anxiety in Portuguese terminally ill patients: A phase II randomized controlled trial. J Palliat Med. 2014; 17: 688-695.

10. Rullán M, Arantzamendi M, Carvajal A, Martínez M, de Ormijana AS, Centeno C. The patient dignity inventory: Just another evaluation tool? Experiences with advanced cancer patients. Palliat Support Care. 2018; 16: 73-79.

11. Rudilla D, Galiana L, Oliver A, Barreto P. Comparing counseling and dignity therapies in home care patients: A pilot study. Palliat Support Care. 2016; 14: 321-329.

12. Martínez $M$, Arantzamendi $M$, Belar A, Carrasco JM, Carvajal A, Rullán $M$, et al. 'Dignity therapy', a promising intervention in palliative care: A comprehensive systematic literature review. Palliat Med. 2017; 31: 492-509.

13. A guide to children's palliative care: Supporting babies, children and young people with lifelimiting and life threatening conditions and their families. Bristol, UK: Together for Short Lives; 2018.

14. Liben S, Papadatou D, Wolfe J. Paediatric palliative care: Challenges and emerging ideas. Lancet. 2008; 371: 852-864.

15. Rodriguez $A$, King $N$. The lived experience of parenting a child with a life-limiting condition: $A$ focus on the mental health realm. Palliat Support Care. 2009; 7: 7-12.

16. Aldridge J, Shimmon K, Miller M, Fraser LK, Wright B. 'I can't tell my child they are dying'. Helping parents have conversations with their child. Arch Dis Child Educ Pract Ed. 2017; 102: 182-187.

17. Rodriguez A, Smith J, McDermid K. Dignity therapy interventions for young people in palliative care: A rapid structured evidence review. Int J Palliat Nurs. 2018; 24: 339-349.

18. Booth A, Maddison J, Wright K, Fraser L, Beresford B. Research prioritisation exercises related to the care of children and young people with life-limiting conditions, their parents and all those who care for them: A systematic scoping review. Palliat Med. 2018; 32: 1552-1566.

19. Akard TF, Dietrich MS, Friedman DL, Hinds PS, Given B, Wray S, et al. Digital storytelling: An innovative legacy-making intervention for children with cancer. Pediatr Blood Cancer. 2015; 62: 658-665.

20. Vaghee S, Javadi RA, Mazlom S, Davoudi N, Gharavi MM, Zare M. The effect of dignity therapy on hope level in patients with chronic renal failure undergoing hemodialysis. Life Sci J. 2012; 9: 3722-3727.

21. Julião $M$, Antunes $B$, Santos A, Sobral MA, Albuquerque $S$, Fareleira $F$, et al. Adapting the Portuguese dignity question framework for adolescents: Ages 10-18. Palliat Support Care. 2019: 1-7.

22. Involve N. Public involvement in research: Values and principles framework. Eastleigh, UK: Nihr Involve; 2015. 
23. Watts $L$, Rodriguez $A$, Tatterton $M$, McSherry W, Smith J. Meaning making and generativity in children and young people with life limiting conditions (MAGICYL). Evid Based Nurs. 2019; 22: 33-35.

24. Shepherd C, Treseder P. Participation: Spice it up! Dynamix, Swansea; 2002.

25. Braun V, Clarke V. Using thematic analysis in psychology. Qual Res Psychol. 2006; 3: 77-101.

26. Foster TL, Dietrich MS, Friedman DL, Gordon JE, Gilmer MJ. National survey of children's hospitals on legacy-making activities. J Palliat Med. 2012; 15: 573-578.

27. Davis TL, Kerr BA, Kurpius SER. Meaning, purpose, and religiosity in at-risk youth: The relationship between anxiety and spirituality. J Psychol Theol. 2003; 31: 356-365.

28. Van Dyke CJ, Elias MJ. How forgiveness, purpose, and religiosity are related to the mental health and well-being of youth: A review of the literature. Ment Health Relig Cult. 2007; 10: 395-415.

29. Johnston B, Lawton S, McCaw C, Law E, Murray J, Gibb J, et al. Living well with dementia: Enhancing dignity and quality of life, using a novel intervention, Dignity Therapy. Int J Older People Nurs. 2016; 11: 107-120.

30. Rodriguez $\mathrm{A}$. We are here for a good time not a long time: Being and caring for a child with a life-limiting condition. University of Huddersfield; 2009.

31. Donovan LA, Wakefield CE, Russell V, Cohn RJ. Hospital-based bereavement services following the death of a child: A mixed study review. Palliat Med. 2015; 29: 193-210.

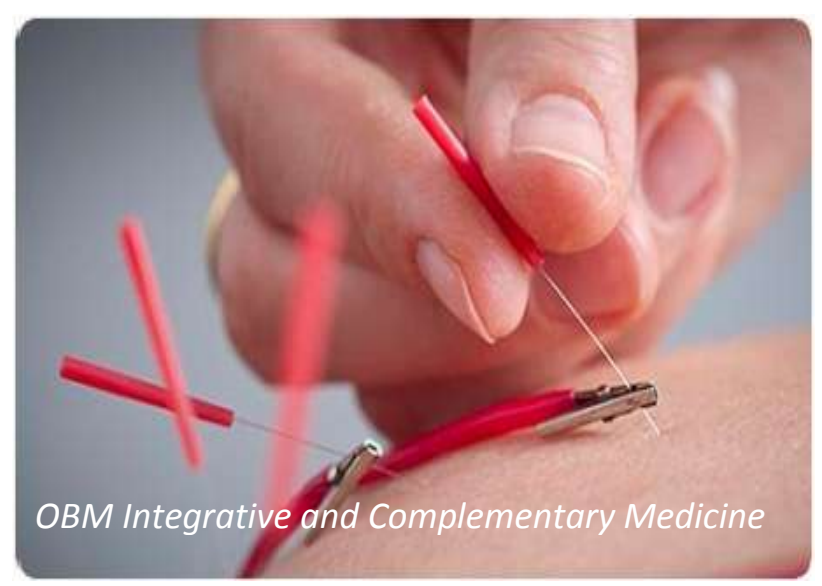

Enjoy OBM Integrative and Complementary Medicine by:

1. Submitting a manuscript

2. Joining in volunteer reviewer bank

3. Joining Editorial Board

4. Guest editing a special issue

For more details, please visit: http://www.lidsen.com/journals/icm 